\title{
Avances en Tecnología de Atmósferas Controladas y sus Aplicaciones en la Industria. Una Revisión
}

\author{
Juan C. De la Vega, Magali A. Cañarejo y Nicolás S. Pinto \\ Universidad Técnica del Norte, Facultad de Ciencias Agropecuarias y Ambientales, Imbabura, Ecuador \\ (e-mail: jcdelavega@utn.edu.ec; macanarejo@utn.edu.ec; nspinto@utn.edu.ec)
}

Recibido Sep. 26, 2016; Aceptado Nov. 28, 2016; Versión final Ene. 25, 2017, Publicado Jun. 2017

\begin{abstract}
Resumen
Este documento describe la historia de la tecnología de atmósferas controladas, sus avances tecnológicos y sus aplicaciones, proporcionando perspectivas de investigaciones en distintos campos de estudio. Para ello se ha desarrollado una revisión de información, sobre los distintos campos donde la tecnología ha sido aplicada y su eficiencia como alternativa de tratamiento. Los estudios relacionados con este campo generados hasta la fecha, están asociados con el efecto que produce la tecnología en las características organolépticas y funcionales de determinadas frutas (aroma, calidad, potencial antioxidante, polifenoles, etc.) y en la automatización de la tecnología (adaptaciones, monitoreo computarizado), así como el efecto en el campo de la electrónica. En la mayoría de los casos la tecnología ha mostrado una mayor eficiencia que en los métodos tradicionales. Se puede concluir que debido a la amplia alternativa de aplicación y la eficiencia de la de la tecnología de atmósferas controladas, se pueden generar estudios adicionales para complementar su desarrollo futuro.
\end{abstract}

\section{Advances in Controlled Atmosphere Technology and its Industrial Applications. A Review}

\begin{abstract}
This document seeks to describe the history of the technology of controlled atmospheres, its technological advances and applications, providing research perspectives in different fields of study. For this purpose, a review of information has been developed, about the different fields where this technology has been applied and its efficiency as an alternative method. The studies related to this field generated until today are associated with the effect that the technology produces on the organoleptic and functional characteristics of certain fruits (aroma, quality, antirust potential, polyphenols) as well as the automation of the technology (adjustments, computerized monitoring), and their applications in the field of electronics. In most cases the technology has shown higher efficiency than the traditional methods. It is possible to conclude that due to the wide alternatives of applications of the technology, additional studies can be generated to complement its future development.
\end{abstract}




\section{INTRODUCCIÓN}

La tecnología de atmósferas controladas se ha desarrollado desde el año 1925 (Yahia, 1995a), por lo cual existe gran información al respecto. Para recopilar los enfoques de los estudios realizados a la fecha se desarrolla el presente trabajo con el fin de establecer una guía sobre los lineamientos de investigación desarrollados y los posibles campos de estudio que se pueden seguir desarrollando. Varios estudios buscan la posibilidad de extender la vida útil de los alimentos durante el almacenamiento, mediante el desarrollo de tecnologías que permitan disminuir pérdidas de productos durante ésta etapa de su vida. Numerosos factores influyen en la descomposición de los alimentos durante el almacenamiento y su estudio puede llegar a ser complejo. Sin embargo una de las tecnologías aplicadas que rigen la distensión del proceso de descomposición de los alimentos son las atmósferas controladas (Barreiro y Sandoval, 2006).

Esta tecnología modifica la concentración de los gases presentes en la atmósfera ambiental, de tal manera que aprovecha su incidencia en la maduración de productos. En los alimentos, esta modificación de la concentración de gases, disminuye la tasa de respiración y provoca un retardo en el tiempo de descomposición. Dicho proceso permite mantener a los productos con posibilidades de ser consumidos, luego de un periodo de tiempo considerable desde que fue producido. Esta característica es de vital importancia cuando se desea por ejemplo, exportar un alimento a tierras lejanas en cantidades considerables, buscando el menor costo posible por envío (Barreiro y Sandoval, 2006).

Otro aspecto importante en el estudio de la conservación de alimentos, refiere a sus características organolépticas y nutritivas. Un alimento con éstas características tan deseables como las que posee en su producción, es un objetivo importante para ofrecer al consumidor. Éstas características están estrechamente relacionadas con la calidad del alimento y su posibilidad de venta y consumo, es por ello que los métodos que se desarrollen en la conservación de alimentos durante su almacenamiento, no sólo deben ser capaces de prolongar el tiempo de vida del alimento, sino también, en lo posible conservar las características sensoriales del fruto.

Lo mencionado anteriormente, refleja los distintos estudios desarrollados, sobre tecnologías de almacenamiento y su efecto sobre las características de los alimentos. Las atmósferas controladas no son la excepción, varios estudios se han realizado en distintos alimentos de diferentes características (Magaña et al., 2006; Salazar y Orozco, 2011), con el objetivo de optimizar, estudiar o describir la relación del proceso y el comportamiento de las características inherentes al alimento de estudio. Sin embargo, esta tecnología no se limita al almacenamiento de alimentos y sus efectos, sino también es aplicada en campos tan poco relacionados como la electrónica. Donde ciertas aplicaciones son alcanzadas con su uso. Entre ellas se encuentra evitar la oxidación del cobre desnudo durante la sinterización de pastas que acoplan dispositivos a su superficie (Zheng et al., 2014), así como el envejecimiento de superficies de materiales dieléctricos, que busca reproducir la química de la arborescencia eléctrica (Freebody y Vaughan, 2010). El efecto de disminución de proliferación de plagas en alimentos también ha sido objeto de estudio. Un claro ejemplo es la mortalidad de Nasonovia Ribisnigri en lechuga (Liu, 2003).

En este trabajo se revisaron distintos estudios asociados a los avances relacionados con la tecnología de atmósferas controladas, destacando sus características positivas y negativas en sus distintas aplicaciones, para describir con mayor amplitud el tema a tratar. El ahorro de energía, monitoreo computarizado de la atmósfera y efectos generados por la aplicación de la atmósfera controlada en determinados alimentos, son algunos de los temas recopilados en este estudio, descritos a través de la historia de la tecnología, su funcionamiento y sus distintas aplicaciones.

\section{TECNOLOGÍAS DE ATMÓSFERAS CONTROLADAS}

En el campo de la preservación de alimentos, generalmente se aplican químicos, plaguicidas y preservantes para mantener sus características por más tiempo, alargar su tiempo de vida útil, y evitar la proliferación de plagas. Ante el progresivo uso de estos productos, que ya se ha evidenciado su efecto negativo en la salud, así como la presencia de residuos en los alimentos (Latham, 2002), surge la necesidad de buscar métodos alternativos de conservación de alimentos. Los métodos físicos de almacenamiento fueron los primeros en ser investigados y desarrollados, como el uso de bajas y altas temperaturas para la conservación de alimentos, así como métodos menos convencionales como atmósferas modificadas, irradiaciones y atmósferas contraladas.

Según Yahia (1995a) el primer trabajo experimental que se desarrolló para establecer las atmósferas controladas como tecnología, se realizó en 1925 por Quid y Est en Inglaterra, quienes descubrieron que el incremento de la concentración del $\mathrm{CO}_{2} \mathrm{o}$ la disminución del $\mathrm{O}_{2}$, generaban un efecto positivo en la conservación de la manzana aumentando su tiempo de vida útil luego de ser cosechada. El uso de la 
atmósfera debe mantenerse durante todo el período de almacenamiento del producto, no sólo por la modificación de la atmósfera sino por la hermeticidad del cuarto en donde es almacenado; esta tecnología retarda la maduración del alimento y evita daños por frío que por lo general también es un factor común en la preservación de alimentos. Gracias a sus beneficios y factibilidad a lo largo del tiempo, la tecnología de atmósferas controladas comenzó a utilizarse para el almacenamiento y transporte de grandes cantidades de alimentos. Hasta la fecha, aproximadamente 3 millones de toneladas de alimentos se almacenaban con atmósferas controladas, debido a su practicidad y buen funcionamiento.

Según lo descrito hasta ahora, la tecnología se trabaja en función a la modificación de la atmósfera en donde va a ser aplicada. En el caso de conservación de alimentos, puede trabajarse a través del control de Dióxido de Carbono generado de la respiración de los alimentos, así como el control de la concentración de $\mathrm{O}_{2}$, para la disminución de la tasa de respiración; o por control de algún otro elemento inherente a la descomposición de los alimentos (como producción de Etileno). Según Yahia (1995a) disminuir la concentración de $\mathrm{O}_{2}$ a valores cercanos al $1 \%$ en la conservación de manzanas, puede influir negativamente en la fruta. Por tanto, es necesario mantener un constante monitoreo de la concentración del $\mathrm{O}_{2}$ en la atmósfera para evitar su declive en el tiempo. Distintos estudios realizados describen la concentración de $\mathrm{O}_{2}$ más eficiente por cada alimento a una determinada temperatura (Barreiro y Sandoval, 2006), y esto a su vez aumenta la eficiencia de la tecnología al puntualizar el proceso para cada fruta u hortaliza. Una posibilidad para controlar el nivel de $\mathrm{O}_{2}$ es introducir aire del ambiente externo, y debido a la respiración del alimento, remover el $\mathrm{CO}_{2}$ para aumentar la eficiencia del proceso. En el caso de atmósfera controlada por $\mathrm{CO}_{2}$, según Yahia (1995a), se somete el producto a un pretratamiento, donde se coloca en contacto con una atmósfera alta en concentración de $\mathrm{CO}_{2}$, por algunas semanas antes de almacenarse en la atmósfera controlada final. Esto permite alargar el tiempo de vida útil del producto, sin embargo este método es poco utilizado.

Durante el proceso de maduración de la fruta, una de las características más relevantes es la producción de etileno. La proliferación de la concentración de este compuesto en el ambiente donde se encuentre almacenada la fruta, acelera el proceso de maduración de la misma. Por tanto mediante el control simultáneo de concentración de $\mathrm{O}_{2}(\approx 2 \%)$ y concentración de $\mathrm{CO}_{2}(<15 \%)$, se puede controlar también la concentración del etileno. Sin embargo, este proceso debe estar constantemente monitoreado, debido a la baja concentración de etileno que debe existir en el ambiente, para evitar la maduración de la fruta, que según Yahia (1995a) debe ser menor a 3 ppm.

En general, una instalación de atmósfera controlada, requiere de techos, pisos y paredes herméticas recubiertas con materiales que permitan asegurar la condición anterior. Así como sistemas de control continuo de concentraciones de gases y de sistemas de control de presión interna, para evitar daños a las instalaciones por una sobre presión o disminución de la misma. Además, se requiere de un sistema de refrigeración para mantener la temperatura a condiciones cercanas a $0^{\circ} \mathrm{C}$ (dependiendo de la fruta) para incrementar la eficiencia de la tecnología. En el caso de aplicaciones electrónicas, las atmósferas se modifican en función principalmente al nivel de oxígeno, con el uso de tratamientos térmicos en determinados casos, para aumentar la eficiencia de la tecnología. El principal objetivo en este campo es lograr establecer un control que logre disminuir las complicaciones generadas por la presencia de oxígeno, que afectan el buen desenvolvimiento del proceso en estudio.

\section{COMPARACIÓN CON OTROS MÉTODOS DE ALMACENAMIENTO}

Al mismo tiempo que se ha desarrollado la tecnología de atmósferas controladas en la conservación de alimentos, distintos métodos se han implementado a nivel industrial para elevar la eficiencia en este campo. Entre los beneficios que posee la tecnología de atmósferas controladas según Barreiro y Sandoval (2006), se encuentran el retardo de la madurez y el envejecimiento, reducción de la sensibilidad al etileno, alivio de daños por frío y control de algunos microorganismos fitopatógenos debido a la reducción de $\mathrm{O}_{2}$. Sin embargo, entre las desventajas se encuentran el desarrollo de malos olores y sabores a causa de producción de alcoholes y aldehídos, provenientes del metabolismo anaeróbico; así como desórdenes fisiológicos como oscurecimiento. Estos últimos inconvenientes están ausentes en técnicas de almacenamiento tradicionales como reducción de temperatura, pero en este caso, se produce daño por frío al producto y a la textura del mismo, por la congelación de moléculas de agua. En esta tecnología puede darse proliferación de plagas si las instalaciones no son completamente herméticas. Una modificación u optimización del almacenamiento por reducción de temperatura es justamente la atmósfera controlada, donde se trabaja con temperaturas cercanas a $0^{\circ} \mathrm{C}$, pero la disminución de la concentración del $\mathrm{O}_{2}$, ayuda a disminuir los efectos negativos de la técnica de almacenamiento tradicional a bajas temperaturas, haciéndola más efectiva.

En el caso de las atmósferas modificadas, los alimentos son sometidos de igual manera a atmósferas diversas a la ambiental dentro de los empaques, en donde se almacena cada fruta o una cantidad determinada de la misma; para ello se desarrolla un material especial (polietileno) para el empacado, con un espesor 
determinado que permita el ingreso de oxígeno y la salida de $\mathrm{CO}_{2}$ y vapor de agua, hasta un punto en donde la concentración de los gases dentro del empaque ralenticen la respiración del alimento. Como alternativa, también se retira el aire del empaque introduciendo gases inertes como Nitrógeno, que disminuyen la concentración inicial de oxígeno y por ende la tasa de respiración del alimento. De esta forma, el crecimiento de los microorganismos disminuye ante la pérdida de oxígeno y el tiempo de vida útil de la fruta aumenta. Sin embargo, el control de ésta atmósfera se realiza únicamente al inicio del almacenamiento, lo que podría ser una desventaja, por cualquier actividad indeseada que se presente posteriormente. Además, por su forma de aplicación, la atmósfera modificada es de bajo costo y es destinada a pequeñas cantidades de alimentos. Posibles desventajas se asocian a daños por reacciones anaeróbicas (Barreiro y Sandoval, 2006). Andrade et al. (2012) reportaron efectos negativos de la atmósfera modificada en el almacenamiento de trozos de ñame, donde se desarrollaron malos olores en algunos tratamientos debido a la respiración anaeróbica del producto, que generó acumulación de etanol y acetaldehído. También se presenció pardeamiento en el alimento por oxidación enzimática que no logró ser controlada en las condiciones estudiadas. En ocasiones este método se trabaja también bajo refrigeración como en el estudio del efecto del tipo de empaque y de corte sobre los cambios físico-químicos y sensoriales de la zanahoria, donde el empaque al vacío generó la conservación más adecuada para la hortaliza estudiada (Dussán-Sarria et al., 2015).

Una última variante de tecnología de conservación de alimento es la aplicación de almacenamiento por baja presión, disminuye la concentración de los gases y mantiene un control bastante exacto en los componentes controlados. Esta tecnología es hermética, no se producen daños por frío en los alimentos, ni cambios en la textura de la fruta. Sin embargo, este último método es bastante costoso por las instalaciones requeridas para mantener las bajas presiones, por lo tanto los implementos que se utilizan para la construcción del espacio de almacenamiento son pesados y especializados. Debido a lo anterior, esta tecnología es poco utilizada, ya que dificulta su uso especialmente en transporte para exportaciones (Yahia, 1995a).

En condiciones de exportación, el almacenamiento por atmósferas controladas suele ser una de las mejores opciones entre las descritas anteriormente, por su capacidad de almacenaje, disminución de daños a la fruta y relativo bajo costo. Lo cual, ha permitido incrementar la aplicación de atmósferas controladas en distintos alimentos, con el fin de conocer el almacenaje óptimo para cada fruta, así como sus posibles efectos en la misma. Los aspectos más relevantes de estudios asociados a las principales aplicaciones de la tecnología de atmósferas controladas tanto en alimentos como en otros ámbitos, se refieren en el siguiente Capítulo. La aplicación más desarrollada es el almacenamiento y conservación de alimentos. Sin embargo, dicha tecnología ha sido aplicada en otras áreas como tratamiento para el control de plagas en alimentos o aplicaciones en electrónica para determinadas actividades. Distintos estudios asociados a las aplicaciones mencionadas, serán descritos a continuación.

\section{ALMACENAMIENTO}

Thompson (2016) menciona que existen muchas variables que afectan la degradación de los alimentos durante su almacenamiento luego de ser cosechados. Algunos de éstas variables refieren a la infección por microorganismos, el ambiente donde se encuentra almacenada, la madurez con que fue cosechada, entre otros. Por lo cual, los requisitos para almacenar la fruta pueden variar, no sólo por lo antes descrito sino porque las frutas tienden a presentar comportamientos diferentes luego de ser cosechadas; generalmente se clasifican en climatéricas o no climatéricas y existen frutas con comportamientos intermedios. Las frutas climatéricas se representan por un crecimiento de la tasa de respiración durante su etapa de descomposición, que se asocia con la producción de etileno. La biosíntesis del etileno genera el incremento de la tasa de respiración apoyado por otros cambios químicos, para el caso de frutas no climatéricas, su composición química se mantiene en el tiempo, a salvo de los azúcares que se descomponen para procesos metabólicos. Lo anterior genera un mayor número de cambios fisiológicos en frutas climatéricas que pueden ser reducidos mediante la manipulación de la atmósfera donde se encuentren almacenadas. Es importante desarrollar los estudios a frutas específicas para conocer el comportamiento de sus componentes químicos asociados a sus características organolépticas, como cambios de color por pérdida de clorofila o síntesis de carotenoides y otros pigmentos. Cambios en las paredes celulares que generan una textura más suave de la fruta, debido a la degradación del almidón en azúcar. El efecto de la atmósfera controlada en los cambios químicos anteriormente descritos son fuente de estudio para conocer su comportamiento, así como el efecto en las bondades nutracéuticas que puedan tener los alimentos sometidos a la tecnología.

Uno de los primeros trabajos asociados al uso de atmósferas controladas en almacenamiento de alimentos, se llevó a cabo con tomates y manzanas. Estos estudios se han extrapolado a otros alimentos como pitahaya, papas, tuna, melones, pepinillos entre otros. Las variables que se estudian en estos trabajos, son la concentración de oxígeno, concentración de dióxido de carbono y temperatura de almacenamiento. Las mismas se han especificado en la literatura para distintos alimentos, según se muestran en la tabla 1. 
En el caso de los efectos producidos en los alimentos a causa de las atmósferas controladas, se puede citar distintas investigaciones. López et al. (2011), indican que el efecto de las atmósferas controladas $\left(4 \mathrm{kPa} \mathrm{O}_{2}+\right.$ $96 \mathrm{kPa} \mathrm{N}$ ) en tomate imperial en estado de madurez comercial, es más efectivo que el ejercido por almacenamiento a baja temperatura. Las variables estudiadas en este trabajo fueron; el color externo, la firmeza, sólidos solubles, producción de $\mathrm{CO}_{2}$ y etileno así como también, el contenido de ácido ascórbico, $\beta$ Caroteno y licopeno.

El factor de mayor relevancia en este estudio refiere al ácido ascórbico por su aporte nutricional, considerado como vitamina para el consumidor. Los resultados muestran que luego de almacenar el tomate en atmósfera controlada durante tres días, y exponerlo a 12 días a $23^{\circ} \mathrm{C}$ a condiciones ambientales, el contenido de ácido ascórbico del tomate almacenado en atmósfera controlada alcanzó un valor de $16.4 \mathrm{mg} / 100 \mathrm{~g}$ PF, mientras que almacenada en temperatura ambiente presentó un valor de $11.7 \mathrm{mg} / 100 \mathrm{~g} P F$. El ácido ascórbico presente en el tomate se degrada a raíz de la enzima ácido ascórbico oxidasa, que actúa en presencia de oxígeno. Los mayores niveles de ácido ascórbico en el tomate almacenado en atmósferas controladas se debe a que se degradaron en menor medida por la ausencia de oxígeno. La provitamina A ( $\beta$-Carotenos) por su parte, presentó valores menores en el alimento al ser retirado de la cámara de almacenamiento que el alimento almacenado con aire. Luego de 12 días sometidos a condiciones ambientales, el tomate almacenado en atmósferas controladas presentó valores mayores de provitamina A. Lo anterior se debe a que, en condiciones reducidas de oxígeno la enzima licopeno $\beta$-ciclasa que cataliza la conversión de licopeno a $\beta$-caroteno, presenta menor actividad por la ausencia de oxígeno, acelerando su activación luego de encontrarse en condiciones normales, lo cual demuestra el efecto positivo que producen las atmósferas controladas en los alimentos.

En otro estudio, Gómez y Camelo (2002) investigaron el color de los tomates almacenados en atmósfera controlada que fueron medidos a través de un colorímetro, utilizando la escala CIEL*a*b. Determinaron que a menor concentración de oxígeno, menor es el cambio en el color durante la maduración del alimento, debido a que hay menor síntesis de licopeno y $\beta$-carotenos, compuestos asociados al color en los alimentos, concordando con el estudio anterior. El menor cambio en la firmeza del tomate almacenado en atmósfera controlada en relación al almacenamiento en aire, está asociado a la disminución de la sintetización de la enzima poligalacturonasa, celulasa y pectinesterasa, que requieren de oxígeno y etileno. Majidi et al. (2014), en un estudio similar, concluyen que en relación a la acidez titulable del tomate, asociada a la disminución de la calidad del alimento, luego de 90 días de almacenado en atmósfera controlada, presenta un valor mayor de acidez titulable, que en 40 días de almacenamiento en refrigeración, superando también el valor obtenido para el almacenamiento del tomate en atmósfera modificada a 90 días.

En manzanas se han hecho estudios adicionales, Salazar y Orozco (2011), realizaron un estudio sobre el efecto de la atmósfera controlada sobre el aroma de la manzana, obteniendo como resultado, que dicha tecnología genera un efecto depresivo en la producción de compuestos volátiles (ácidos, ésteres, alcoholes y aldehídos) asociados al aroma de la fruta, debido a que estos son generados por la degradación oxidativa del ácido linoléico y linolénico. Calu et al. (2009) estudiaron las propiedades fisicoquímicas de la manzana durante el almacenamiento con atmósferas controladas, en comparación con refrigeración tradicional. Los resultados mostraron que luego de 8 meses de almacenamiento en todas las variedades de manzanas sometidas a atmósferas controladas se evidenció contenido de vitamina $\mathrm{C}$, no así para el caso de las manzanas sometidas a refrigeración. En otro estudio, Duroňová et al. (2012) investigaron el efecto del almacenamiento a largo plazo en atmósferas controladas sobre el contenido de ácidos grasos y lípidos en las células de manzana. Encontraron que entre los principales ácidos grasos presentes en la fruta, se encuentran el ácido linoleico y el ácido a-linoleico, los cuales son ácidos esenciales para la dieta humana. Se encontró que tanto en la pulpa como en la piel de la manzana la concentración de los ácidos grasos luego de 4 meses de almacenamiento era poco más del doble en las frutas almacenadas en atmósferas controladas en referencia a las manzanas almacenadas en condiciones de refrigeración. A los 6 meses de almacenamiento el contenido de ácido a-linoleico en la pulpa de la manzana decreció drásticamente en el fruto almacenado en atmósfera controlada, por lo cual no se logró ubicar una descripción predecible de la degradación del ácido en las condiciones de almacenamiento. Wang et al. (2011), por su parte estudiaron los efectos de la tecnología en melones, analizando los componentes aromáticos de dicha fruta, en atmósferas con niveles de oxígeno entre $4 \%$ y $6 \%$, y niveles de dióxido entre $0.2 \%$ y $0.5 \%$. Obtuvieron que las atmósferas controladas mantenían en mayor medida los componentes asociados a su aroma, en especial los ésteres. En atmósfera controlada se obtuvo un $85.49 \%$ de presencia de ésteres, mientras que en la refrigeración normal se obtuvo un $62.41 \%$. Guo et al. (2013) enfocaron sus estudios en el efecto de la atmósfera controlada sobre brócolis, al evaluar la capacidad antioxidante que presenta el alimento después de ser almacenado en estas condiciones, a través de la medición de la actividad de las enzimas superóxido dismutasa y peroxidasa. Trabajaron con atmósferas con exceso de oxígeno y $\mathrm{CO}_{2}$, determinando que a concentraciones de $40 \%$ de oxígeno y $60 \%$ de $\mathrm{CO}_{2}$, existe una estabilidad en la actividad de estas enzimas, evitando que las células del alimento puedan verse afectadas por oxidación. En casos extremos como la exposición del alimento a una atmósfera de $100 \%$ de 
oxígeno, se evidenció un aumento drástico en la actividad de las enzimas los primeros días de almacenamiento, pero que decayó luego de esto, lo cual se debe a la alta exposición de oxígeno que sufre el alimento, incentivando la actividad de las enzimas, pero ante la continua exposición a altas concentraciones de oxígeno no son capaces de mantener su actividad. A través de este estudio se evidencia también la posibilidad de almacenar frutas y hortalizas en atmósferas extremas, lo cual no necesariamente puede ser beneficioso para todas las frutas, pues en esta revisión se ha evidenciado que no todas poseen el mismo comportamiento.

Tabla 1: Condiciones de almacenamiento en atmósferas controladas de algunos productos (Barreiro y Sandoval, 2006)

\begin{tabular}{|c|c|c|c|}
\hline Alimento & Temperatura $\left({ }^{\circ} \mathrm{C}\right)$ & Oxígeno (\%) & $\mathrm{CO}_{2}(\%)$ \\
\hline Aceituna & $8-12$ & $2-5$ & $5-10$ \\
\hline Aguacate & $5-13$ & $2-5$ & $5-10$ \\
\hline Albaricoque & $0-5$ & $2-3$ & $2-3$ \\
\hline Banana Cavendish Madura & 15 & 2 & $6-8$ \\
\hline Banana Cavendish/Enana/Gran enana verde & 15 & 2 & $6-8$ \\
\hline Banana Gros Michel & 12 & 5 & 5 \\
\hline Banana Latundan Madura & 15 & 2 & $6-8$ \\
\hline Banana Lacatan Verde & 15 & 2 & $6-8$ \\
\hline Banana Latundan Verde & 15 & 2 & $6-8$ \\
\hline Banana Poovan Verde & 15 & 2 & $6-8$ \\
\hline Cereza & $0-5$ & $0-5$ & $10-12$ \\
\hline Ciruela & $0-5$ & $1-2$ & $0-5$ \\
\hline Durazno, Melocotón & $0-5$ & $1-2$ & 5 \\
\hline Fresa & $0-5$ & 10 & $15-20$ \\
\hline Higo & $0-5$ & 5 & 15 \\
\hline Kaki, Caqui & $0-5$ & $3-5$ & $1-8$ \\
\hline Kiwi & $0-5$ & 2 & 5 \\
\hline Lechuga & $0,5-1$ & $2-5$ & $<2$ \\
\hline Lima & $10-15$ & 5 & $0-10$ \\
\hline Limón Verde & $10-15$ & 5 & $0-5$ \\
\hline Mango & $8-15$ & $5-10$ & $5-6$ \\
\hline Manzana & $0-5$ & $2-3$ & $1-2$ \\
\hline Melón Cantaloupe & $5-10$ & $3-5$ & $10-15$ \\
\hline Melón Honeydew Honey ball & $10-12$ & $3-5$ & 0 \\
\hline Naranja Dulce & $1-10$ & $10-15$ & $0-7$ \\
\hline Papaya, Lechosa & $10-15$ & $10-15$ & $0-7$ \\
\hline Pera & $0-5$ & $2-3$ & $0-1$ \\
\hline Pimiento dulce/ ají/ Paprika & $8-12$ & $3-5$ & $0-10$ \\
\hline Piña Verde & $10-15$ & 5 & 10 \\
\hline Tomate Maduro Pintón & $8-12$ & $3-5$ & 0 \\
\hline Tomate Verde & $12-20$ & $3-5$ & 0 \\
\hline Toronja & $10-15$ & $3-10$ & $5-10$ \\
\hline
\end{tabular}

Ozer et al. (2006), realizaron sus estudios enfocados en pepinillos, para verificar la posibilidad de extender el tiempo de almacenamiento del pepino antes de ser procesado a pepinillo. El principal inconveniente resulta en que el tiempo de almacenamiento debe evitarse para que no afecte las propiedades físicas, químicas y sensoriales del producto final. En este estudio se encontró que al igual que en los estudios en tomates, la reducción del oxígeno genera un efecto positivo en la firmeza del pepino, asociado a que hay menor pérdida de peso, debido a la menor pérdida de agua. Lo anterior se produce en parte por la baja actividad metabólica debido a la ausencia de oxígeno, añadiendo el efecto de la inhibición de la destrucción enzimática de los polisacáridos, la pectina y la celulosa. Alba (2014), estudió el efecto de la atmósfera controlada sobre la actividad antioxidante de la tuna roja, encontrando que gracias a esta tecnología luego de 16 días de almacenamiento, la fruta mantiene su poder antioxidante ante la conservación de contenido de polifenoles y betalaínas. Estos compuestos pueden servir como protectores debido a su actividad antioxidante, ya que regulan los radicales libres presentes en el cuerpo humano limitando así la probabilidad de generación de 
cáncer. En el estudio se encontró que a menor concentración de oxígeno la cantidad de polifenoles es mayor luego de 16 días de almacenamiento, en relación a atmósferas con mayor contenido de oxígeno. Esto debido al daño mecánico generado por la oxidación en los compuestos fenólicos. Las betalaínas por su parte presentaron mayor concentración en atmósferas con baja concentración de oxígeno y alta concentración de $\mathrm{CO} 2$, debido a que en estas condiciones la actividad de algunas enzimas como la peroxidasa se ve disminuida, logrando obtener un alimento con propiedades nutricionales cercanas al momento de su cosecha, luego de un período de almacenamiento. Según Sánchez et al. (2007), se ha desarrollado poca investigación sobre la capacidad antioxidante de frutas tropicales en función a un período de almacenamiento, sobre todo en frutos frescos cortados. Este campo de investigación está susceptible a desarrollarse para generar conocimiento sobre las posibles bondades que puede crear la tecnología de atmósferas controladas.

Gökmen et al. (2007) estudiaron los efectos de la atmósfera controlada en dos variedades de papas (Agria y Russet Burbank), en donde la pérdida de firmeza del tubérculo se limita con la aplicación de la atmósfera controlada. Sin embargo, lo anterior se produce a costa de la liberación de azúcares, lo cual afecta el color de la papa en su procesamiento como fritura, generando en este caso un resultado negativo en las características del tubérculo. En la pitahaya y en el durazno, se han desarrollado estudios asociados sobre los efectos de la atmósfera controlada. Magaña et al. (2006) estudiaron el efecto de la atmósfera controlada en la pitahaya, encontrando que a menores concentraciones de oxígeno se genera una tasa mayor de etanol y acetaldehído, debido a la respiración anaerobia ocasionando un mal sabor y olor a la fruta. Brackmann et al. (2009) encontraron que al igual que en la mayoría de las frutas, el efecto de la atmósfera controlada es más eficiente que el método tradicional por baja temperatura, en cuanto al aumento del tiempo de vida útil.

Frutas exóticas como Fuyu Persimmon, también han sido estudiadas con atmósferas controladas en distintas combinaciones de concentraciones de $\mathrm{O}_{2}$ y CO 2 . Brackmann et al. (2014), encontraron que la atmósfera con $0.15 \mathrm{kPa} \mathrm{O}_{2}$ y $6 \mathrm{kPa} \mathrm{CO} 2$ presentó el mejor efecto sobre la conservación de variables asociadas a la descomposición de la fruta (sólidos solubles, tasa de respiración, pérdida de peso, cociente respiratorio). En este estudio se comprobó que el aumento en la presión parcial del $\mathrm{CO}_{2}$ limita la tasa de respiración de la fruta, debido a que se reduce la producción de $\mathrm{CO}_{2}$ generado en el ciclo ácido tricarboxílico, afectando en menor medida la pérdida de masa a causa de la respiración de la fruta. En el caso de cerezas Laetitia, Steffens et al. (2014), estudiaron el efecto de la tecnología de atmósferas controladas sobre la descomposición de la fruta luego del almacenamiento. Tras someter a la fruta en distintas atmósferas por 60 días, determinaron sus características pasados 4 días fuera del almacenamiento, encontrando que atmósferas con composiciones de $2 \mathrm{kPa} \mathrm{O}_{2}+5 \mathrm{kPa} \mathrm{CO} 2$ y $1 \mathrm{kPa} \mathrm{O}_{2}+3 \mathrm{kPa} \mathrm{CO}_{2}$, resultaron en un atraso sustancial de la maduración de la fruta, así como una menor incidencia en la descomposición interna y bajas tasas de producción de etileno y respiración. En este estudio se verificó que la acidez titulable de la fruta se ve afectada en menor medida en condiciones de atmósfera controlada, ya que existe menor consumo de ácidos orgánicos como fuente de energía para el proceso de respiración.

Saxena et al. (2011) reportaron estudios en la yaca, donde estudiaron el efecto de la atmósfera controlada a diferentes concentraciones de oxígeno y dióxido de carbono en el contenido de fenoles totales. Encontraron que a mayor concentración de dióxido de carbono y baja concentración de oxígeno, se retiene en mayor medida los fenoles totales que en condiciones diferentes. En este estudio utilizaron capas antioxidantes para preservar las propiedades nutracéuticas de la fruta, sin embargo a pesar de esto, se logró determinar la relación entre el uso de atmósferas controladas y la retención de los fenoles totales de la fruta, a causa de la baja disponibilidad de oxígeno molecular.

En crustáceos como camarones, también se han desarrollado investigaciones de almacenamiento bajo atmósferas controladas. En este caso particular, la variable en estudio fue la melanosis en la piel del crustáceo. Se utilizaron atmósferas con $44-46 \%$ de $\mathrm{CO}_{2}$ y el uso de $0.1 \%$-Hexylresorcinol, generó un índice de melanosis por debajo del $2 \%$, alrededor de la mitad encontrado bajo condiciones de almacenamiento normales (Martínez, 2005). En el campo de la utilización de atmósferas controladas en el almacenamiento de alimentos, existen innumerables estudios, que abarcan distintos tópicos. Sin embargo varios estudios pueden seguir desarrollándose en este sentido, aumentando el conocimiento sobre el tema. Adicionalmente las atmósferas controladas también se utilizan en otras aplicaciones como se describirán en las siguientes secciones.

\section{CONTROL DE PLAGAS}

La característica anaeróbica de la tecnología de atmósferas controladas ha sido aprovechada en distintos alimentos y plantas para el control de plagas recurrentes durante su almacenamiento. El beneficio adicional de la aplicación de esta tecnología en el control de plagas, radica en que no hay necesidad de aplicar elementos químicos que puedan dejar residuos tóxicos en el alimento (Yahia, 1995b). Neven (2005) estudió la aplicación de atmósferas controladas en las cerezas para controlar la proliferación de polillas durante su 
almacenamiento, la fruta se procesó en un ambiente escaso de oxígeno (1\%) a una temperatura de $47^{\circ} \mathrm{C}$, durante 25 minutos, obteniendo una mortalidad de larvas de polillas en su tercera fase del 100\%. Se determinó que el principal factor en la mortandad de las larvas es la reducción del nivel de oxígeno, asegurando así la cuarentena de la fruta, sobre todo para fines de exportación.

Son et al. (2012), desarrollaron una investigación parecida en manzanas, para controlar el crecimiento de polillas de gusanos (Lepidoptera: Carposinidae). La fruta fue sometida durante 60 minutos a temperaturas de $46{ }^{\circ} \mathrm{C}$, en una atmósfera de $1 \% \mathrm{O}_{2}$ y $15 \% \mathrm{CO}_{2}$, obteniendo un $100 \%$ de mortalidad desde el primer al quinto estado de las larvas, sin presentar pérdidas de calidad en la fruta en relación a su firmeza o dulzura. Según Neven (2008), los insectos en general regulan la respiración en función al nivel de $\mathrm{CO}_{2}$. En el caso de las atmósferas controladas donde existen altas concentraciones de $\mathrm{CO}_{2}$, los mecanismos normales se inhiben, lo que causa una reducción de ATP, por la reducción del pH, causado a su vez por la generación de ácido carbónico. Lo que ayuda a desestabilizar membranas, generando una ruptura en la fosforilación oxidativa y en el transporte de electrones, disminuyendo así la energía necesaria para el crecimiento de las células de las larvas y degradando el poder evasivo al daño por estrés térmico. De esta forma en la mayoría de los tratamientos realizados con atmósferas controladas en adición a tratamientos térmicos en alimentos, se produce una mortandad de insectos del $100 \%$.

Otra alternativa para el control de plagas en alimentos, es la atmósfera de vacío. Liu (2003), estudió esta alternativa en lechugas Lettuce, para tres tipos distintos de insectos. La eficiencia encontrada en los tratamientos en estudio no es tan alta como la indicada en los estudios anteriormente citados, sin embargo para Liriomyza langei, con $6 \%$ de $\mathrm{CO}_{2}$, a 50 mbar y $5{ }^{\circ} \mathrm{C}$ durante 4 días, consiguió una mortandad superior al $96 \%$. El uso de ésta tecnología además de los alimentos, se podría aplicar en flores y plantas, Hollingsworth y Armstrong (2005), estudiaron la aplicación combinada de atmósferas controladas, con vacío, tratamiento térmico y fumigación con ozono en distintas flores para el control de Frankliniella occidentalis y Pseudococcus longispinus. En éste estudio, se determinó un mayor efecto en la primera plaga (98\% mortandad) que en la segunda (47,9\%) para el mejor tratamiento, demostrando la posibilidad de aplicar la tecnología para distintas flores. Al igual que para los alimentos, la aplicación de esta tecnología resulta de gran utilidad en la comercialización de flores para exportación, donde el objetivo primordial es mantener las características sensoriales y/o físicas, en un período de tiempo prolongado.

\section{APLICACIONES ELECTRÓNICAS}

En un área completamente distinta a lo revisado en las secciones anteriores, la tecnología de atmósferas controladas puede ser aplicada para cumplir con determinados objetivos en la electrónica. Zheng et al. (2014) desarrollaron un estudio en unión de chips sobre cobre mediante sinterización al vacío de pasta nano plateada bajo atmósfera controlada. La sinterización es un proceso donde el material se somete a un tratamiento térmico para aumentar su resistencia mecánica. Para ello se utilizó éste proceso en conjunto con atmósferas controladas, para mejorar la unión de chips sobre cobre. La pasta nano plateada, consta de nanopartículas de plata mezcladas con compuestos orgánicos y aglutinantes, que sirven como un elemento de unión entre superficies luego de un proceso de sinterización, que puede soportar fuerzas superiores a 40 MPa, incluso a altas temperaturas. Estas características son de especial importancia en equipos electrónicos que son sometidos a temperaturas elevadas y a esfuerzo mecánicos extremos. El principal inconveniente en el proceso estudiado es que para sinterizar la pasta nano plateada se requiere de temperaturas cercanas a los $260^{\circ} \mathrm{C}$, en las cuales los elementos de cobre desnudo generan una capa de óxido de cobre, la cual evita la unión entre la pasta, el cobre y el chip. Disminuyendo así, la fuerza de unión a valores menores a 5 MPa. Es por ello la importancia de la aplicación de atmósferas controladas en este campo, para que el oxígeno no reaccione con el cobre formando las capas de óxido. Sin embargo, no es posible remover todo el oxígeno en este proceso, ya que los aglutinantes y los compuestos orgánicos de la pasta nanoplateada requieren ser removidos a través de procesos de combustión en presencia de oxígeno para que se genere efectivamente la aglutinación de la pasta necesaria para el proceso de sinterización. A través de un microscopio electrónico de barrido, se analizaron las imágenes de la unión de la pasta sometida a las distintas atmósferas, logrando verificar que en el caso de la atmósfera con un $1 \%$ de oxígeno, la pasta logra aglutinarse, no obstante, la oxidación del cobre supera la posibilidad de la sinterización de soportar esfuerzos mecánicos. Se constató que con una concentración de $4 \%$ de hidrógeno y el resto de Nitrógeno, se logra una mayor resistencia al esfuerzo mecánico, debido a que la presencia del hidrógeno y las altas temperaturas de trabajo, generan la hidrólisis de los aglutinantes presentes en la pasta, permitiendo a las nanopartículas tener contacto y aumentar su densidad, mejorando así la resistencia al esfuerzo mecánico. Los resultados muestran que la atmósfera controlada afecta considerablemente en el proceso de unión de chips sobre cobre.

En un estudio similar se refleja la aplicación de las atmósferas controladas en el envejecimiento de la superficie de materiales dieléctricos. Freebody y Vaughan (2010), estudiaron esta aplicación para lograr desarrollar un método aproximado, capaz de describir los procesos involucrados en la arborescencia eléctrica, 
como una alternativa ante la ineficiente descripción mediante otros métodos. El envejecimiento de la superficie de materiales dieléctricos, se genera por el estrés eléctrico al cuál se someten. Este envejecimiento produce una ruptura en el material, provocando un deterioro en su funcionamiento, estableciendo la importancia de entender como ocurre este proceso, para poder mitigarlo. Éste estudio se realizó mediante espectroscopía Raman, la caracterización química de distintos dieléctricos antes y después de someterlos a una descarga corona (proceso de envejecimiento en la superficie del material), para establecer la similitud del fenómeno a la arborescencia eléctrica (proceso de envejecimiento en el interior del material). Con el estudio, se identificó que en presencia de atmósferas controladas con completa ausencia del aire, existe una similitud entre los fenómenos mencionados anteriormente, donde se evidencia por los datos obtenidos de la espectroscopía, que existen cambios químicos en el material durante el envejecimiento. Dada la importancia e integridad en la aplicación de la tecnología de atmósferas controladas, se han desarrollado estudios adicionales para mejorar su funcionamiento. La aplicación de nuevos sensores, los estudios de sostenibilidad de la tecnología y la viabilidad de aplicación en nuevos proyectos son algunos tópicos casos de estudio. En la siguiente sección se desarrolla parte de estas investigaciones.

\section{MEJORAS DESARROLLADAS}

La optimización de la tecnología buscando ampliar el campo de aplicación, es uno de los aspectos de estudio que concierne a la mejora de desarrollo. Lin et al. (2013), estudiaron la aplicación de una membrana hueca, en lugar de la adición de Nitrógeno, o la generación de $\mathrm{CO}_{2}$, como alternativa para la modificación de la atmósfera requerida por la tecnología. En dicho estudio se desarrolló un modelo matemático para describir la permeabilidad de la mezcla de gases a través de la membrana, para alcanzar las concentraciones requeridas en la tecnología. Por otro lado Markarian et al. (2003) estudiaron la aplicación de un sistema computarizado para el monitoreo de instalaciones de almacenamiento bajo atmósferas controladas, para ello se desarrolló un controlador tipo proporcional integral ajustando la ganancia Kc, para caracterizar la dinámica del sistema. Como resultado, se encontró errores del 0.0035 y $0.0016 \%$ al punto fijado de referencia para la concentración de $\mathrm{CO}_{2}$ y $\mathrm{O}_{2}$ respectivamente.

East et al. (2013) lograron describir un método para disminuir los costos energéticos asociados a la tecnología, mediante la manipulación de la temperatura de almacenamiento, estimaron hasta un 40\% de reducción en costos energéticos sin generar un efecto significativo en la calidad de la fruta en estudio (manzana). Existen varias posibilidades de mejora o adaptación de la tecnología para ajustar su uso a determinadas aplicaciones. Por ende el campo de estudio en esta área sigue siendo amplio para desarrollar conocimiento e investigaciones.

\section{CONCLUSIONES}

La tecnología de atmósferas controladas posee una aplicación amplia, desde su utilización en la extensión del tiempo de anaquel de distintos alimentos, hasta la unión de chips electrónicos en cobre. Su eficiencia en cada una de sus aplicaciones ha sido estudiada en distintas investigaciones obteniendo resultados prácticos y en general con mayores beneficios que los respectivos métodos convencionales. Sin embargo, aunque su estudio a lo largo del tiempo desde su primera aplicación, se ha incrementado, existen distintas aristas de estudio asociadas a esta tecnología que pueden ser fuente de nuevas investigaciones (estudio del efecto en actividad antioxidante y polifenoles en distintas frutas, mejoramiento de automatización de la tecnología o aplicación en otras áreas electrónicas no estudiadas a la fecha) en función de generar mayor valor agregado y de aprovechar la alta eficiencia de la tecnología. Además, varias aplicaciones y resultados se mencionaron, permitiendo presentar una amplia descripción de la tecnología al momento de realizar el presente trabajo.

\section{REFERENCIAS}

Alba, J., Betalaínas, Polifenoles y Actividad Antioxidante en Tuna Roja Mínimamente Procesada, Almacenada en Atmósferas Controladas, doi:10.4067/S0717-66432014000200005, Gayana Bot., 71(2), $222-226$ (2014)

Andrade, R., J. Palacio, W. Pacheco y R. Betin, Almacenamiento de Trozos de Ñame (Dioscorea rotundata Poir) en Atmósferas Modificadas, doi: 10.4067/S0718-07642012000400008, Inf. Tecnol., 23(4), 65-72 (2012)

Barreiro, J. y A. Sandoval, Operaciones de Conservación de Alimentos por Bajas Temperaturas, $1^{\text {a }}$ edición, 100-101. Equinoccio, Caracas, Venezuela (2006)

Brackmann A., E. Pivotto, V. Both, A. Weber y J. Vilela, Cold and controlled atmosphere of 'Eragil' peaches, doi: 10.1590/S0103-84782009005000149, Ciência Rural, 39(7), 2010-2015 (2009) 
Brackmann A., F. Rodrigo, R. de Oliveira, V. Both y A. Roque, Respiration Rate and its Effect on Mass Loss and Chemical Qualities of 'Fuyu' Persimmon Fruit Stored in Controlled Atmosphere, doi: 10.1590/S010384782014000400006, Ciência Rural, 44(4), 612-615 (2014)

Calu M., C. Bonciu e I. Tofan, Physico-chemical Characteristics of Apples Stored at Chilling and Controlled Atmosphere Conditions, International Symposium Euro-aliment, ISSN: 2068-259X (en línea) 32-37, 34(1), 2009. https://goo.gl/WXsw3O. Acceso: 2 de Mayo (2016)

Duroňová K., I. Márová, M. Čertík y S. Obruča, Changes in lipid composition of apple surface layer during long-term storage in controlled atmosphere, doi: 10.2478/s11696-012-0200-0, Chemical Papers, 66(10), 940$948(2012)$

Dussán-Sarria S., C. Garcia-Mogollon y C. Gutiérrez-Guzmán, Cambios Físico-Químicos y Sensoriales Producidos por el Tipo de Corte y Empaque en Zanahoria (Daucus carota L.) Mínimamente Procesada, doi: 10.4067/S0718-07642015000300010, Inf. Tecnol., 26(3), 63-70 (2015)

East R., N. Smale y F. Trujillo, Potential for Energy Cost Savings by Utilising Alternative Temperature Control Strategies for Controlled Atmosphere Stored Apples, doi: 10.1016/j.ijrefrig.2012.10.028, International J. of Refrigeration, 36(3), 1109-1117 (2013)

Freebody N.A. y Vaughan A.S., Surface Ageing of Dielectric Materials in a Controlled Atmosphere: a Raman Microprobe Study, IEEE Annual Report Conference on Electrical Insulation and Dielectric Phenomena, 1-4, West Lafayette, USA, 17 a 20 de Octubre (2010)

Gómez P.A. y A.F. Camelo, Calidad Postcosecha de Tomates Almacenados en Atmósferas Controladas, doi:10.1590/S0102-05362002000100007, Horticultura Brasileira, 20(1), 38-43 (2002)

Gökmen V., B. Akbudak, A. Serpen, J. Acar, Z.M. Turan y A Eriş, Effects of Controlled Atmosphere Storage and Low-dose Irradiation on Potato Tuber Components Affecting Acrylamide and Color Formations Upon Frying, doi: 10.1007/s00217-006-0357-2, Eur. Food Res. Technol., 224(6), 681-687 (2007)

Guo Y., Z. Gao, L. Li, Y. Wang, H. Zhao, M. Hu, M. Li y Z. Zhang, Effect of controlled atmospheres with varying $\mathrm{O}_{2} / \mathrm{CO}_{2}$ levels on the postharvest senescence and quality of broccoli (Brassica oleracea L. var. italica) florets, doi: 10.1007/s00217-013-2064-0, Eur. Food. Res. Technol., 237(6), 943-950 (2013)

Hollingsworth R.G. y Armstrong J.W., Potential of Temperature, Controlled Atmospheres, and Ozone Fumigation to Control Thrips and Mealybugs on Ornamental Plants for Export, doi: 10.1603/0022-049398.2.289, J. Econ. Entomol., 98(2), 289-298 (2005)

Latham M., Mejoramiento de la calidad y seguridad de los alimentos in Nutrición Humana en el mundo en desarrollo by Colección FAO: Alimentación y Nutrición № 29, pp 353-354, Roma, Italia (2002)

Lin K.C. N. Peng, H. Yin, G.G. Lipscomb y T.S. Chung, Food Sustainability by Designing and Modeling a Membrane Controlled Atmosphere Storage System, doi: 10.1016/j.jfoodeng.2012.08.027, J. Food Eng. 114(3), 361-374 (2013)

Liu Y.B., Effects of Vacuum and Controlled Atmosphere Treatments on Insect Mortality and Lettuce Quality, doi: 10.1603/0022-0493-96.4.1100, J. Econ. Entomol., 96(4), 1100-1107 (2003)

López J.A., F.J. Valverde, S.L. Mejía, G. López y M.O. Vega, Efecto del Almacenamiento en Atmósfera Controlada sobre la Calidad Poscosecha y Nutricional del Tomate, Chapingo Serie Horticultura, ISSN: 1027152X (en línea) 17(2), 115-128, 2011. https://goo.gl/S2dt2V. Acceso: 20 de Abril (2016)

Magaña W., M. Balbín, J. Corrales, A. Rodríguez y C. Saucedo, Principales características de calidad de las pitahayas (hylocereus undatushaworth), frigoconservadas en atmósferas controladas, Ciencias Técnicas Agropecuarias, ISSN: 1010-2760 (en línea), 15(2), 52-57, 2006. https://goo.gl/jqA5N3. Acceso: 10 de Abril (2016)

Majidi H., S. Minaei, M. Almassi y Y. Mostofi, Tomato Quality in Controlled Atmosphere Storage, Modified Atmosphere Packaging and Cold Storage, doi: 10.1007/s13197-012-0721-0, J. Food Sci. Technol., 51(9), 2155-2161 (2014)

Markarian N.R., C. Vigneault, Y. Gariepy y T.J. Rennie, Computerized monitoring and control for a research controlled-atmosphere storage facility, doi: 10.1016/S0168-1699(03)00005-X, Computers \& Electronics in Agriculture, 39(1), 23-37 (2003) 
Martínez O., P. Montero y M.C. Gómez, Controlled Atmosphere as Coadjuvant to Chilled Storage for Prevention of Melanosis in Shrimps (Parapenaeus longirostris), doi: 10.1007/s00217-004-1015-1, Eur. Food Res. Technol., 220(2), 125-130 (2005)

Neven L.G., Combined Heat and Controlled Atmosphere Quarantine Treatments for Control of Codling Moth in Sweet Cherries, doi: 10.1603/0022-0493-98.3.7009, J. Econ. Entomol., 98(3), 709-715 (2005)

Neven L.G., Development of a Model System for Rapid Assessment of Insect Mortality in Heated Controlled Atmosphere Quarantine Treatment, doi: 10.1603/0022-0493(2008)101[295:DOAMSF]2.0.CO;2, J. Econ. Entomol., 101(2), 295-310 (2008)

Ozer M.H., B. Akbudak, V. Uylaser y E. Tamer, The Effect of Controlled Atmosphere Storage on Pickle Production from Pickling Cucumbers cv. 'Troy', doi: 10.1007/s00217-005-0092-0, Eur. Food Res. Technol., 222(1), 118-129 (2006)

Salazar N.S. y G.O. Orozco, El Aroma de la Manzana, Interciencia, ISSN: 0378-1844 (en línea), 36(4), 265271, 2011. http://www.redalyc.org/articulo.oa?id=33917994004. Acceso: 2 de Mayo (2016)

Sánchez M., S. Gorinstein, O. Martín, H. Astiazarán, G. González y R. Cruz, Frutos Tropicales Mínimamente Procesados: Potencial Antioxidante y su Impacto en la Salud, Interciencia, ISSN: 0378-1844, 32(4), 227-232, 2007. https://goo.gl/EkN4nl. Acceso: 15 de Abril (2016)

Saxena A., T. Saxena, P. Raju y A. Bawa, Effect of Controlled Atmosphere Storage and Chitosan Coating on Quality of Fresh-Cut Jackfruit Bulbs, doi: 10.1007/s11947-011-0761-x, Food Bioprocess Technol., 6(8), 21822189 (2013)

Son Y., I. Chon, L. Neven y Y. Kim, Controlled Atmosphere and Temperature Treatment System to Desinfest Fruit Moth, Carposina Sasakii (Lepidoptera: Carposinidae) on Apples, doi: 10.1603/EC12133, J. Econ. Entomol., 105(5), 1540-1547 (2012)

Steffens C.A., C.V. Talamini, E. de Oliveira, A. Brackmann, T.R. Corrêa y B. Pansera, Storage of 'Laetitia' Plums (Prunus Salicina) Under Controlled Atmosphere Conditions, doi: 10.5897/AJB2014.13845, Afr. J. Biotechnol., 13(32), 3239-3243 (2014)

Wang W., C. Chen, J. Ning y H. Wang, The Effect of Controlled Atmosphere Storage on Aroma Components of Hami Melon, International Conference on New Technology of Agricultural Engineering, 764-768, Zibo, China, 27 a 29 de Mayo (2011)

Yahia E., La Tecnología de las Atmósferas Modificadas y Controladas I Parte, Horticultura Internacional, ISSN:1132-2950, (7), 37-39, 1995a. https://goo.gl/189snU. Acceso 13 de enero (2016)

Yahia E., La Tecnología de las Atmósferas Modificadas y Controladas II Parte, Horticultura Internacional, ISSN:1132-2950, (8), 20-25, 1995b. https://goo.gl/4QDeUW. Acceso: 13 de enero (2016)

Zheng H., D. Barry, K.D.T. Ngo y G.Q. Lu, Chip-Bonding on Copper by Pressureless Sintering of Nanosilver Paste Under Controlled Atmosphere, doi: 10.1109/TCPMT.2013.2296882, IEEE Trans. On Components, Packaging and Manufacturing Technology (en línea), 4(3), 377-384 (2014) 
